\title{
Efficacy of Frequency-dependent Conduction Blockade of the Inferior Alveolar Nerve (IAN) for Obtaining Pulpal Anaesthesia: A Prospective Study
}

Dr. Jyoti Kiran ${ }^{1 *}$, Dr. Pratiksha A Srivastava ${ }^{2}$, Dr. Radhika Gupta ${ }^{2}$, Dr. Vishesh Dhir ${ }^{3}$, Dr. Kumar Ravishankar ${ }^{3}$, Dr. Mamta Singh ${ }^{4}$

\author{
${ }^{1}$ (MDS), Reader, Department of Oral and Maxillofacial Surgery, BRS Dental College and Hospital, Sultanpur Panchkula, Haryana, \\ India \\ ${ }^{2}$ (MDS), Third Year Postgraduate Student, Department of Prosthodontics and Crown and Bridge, Subharti Dental College, Swami \\ Vivekanand Subharti University, Meerut, U.P. India \\ ${ }^{3}$ (MDS), Senior Lecturer, Department of Periodontics, Bhojia Dental College and Hospital, Dental school in Baddi, Himachal Pradesh, \\ India \\ ${ }^{4}$ (MDS), Assistant Professor, Department of Periodontics, Kothiwal Dental College and Research Centre, Mora Mustaqueem, Kanth \\ Road, Moradabad, 244001, India
}

DOI: $\underline{10.36347 / \text { sjds.2021.v08i03.001 }}$

| Received: 17.02.2021 | Accepted: 01.03.2021 | Published: 03.03.2021

*Corresponding author: Dr. Jyoti Kiran

Abstract

Original Research Article

Aim: The present randomized single-blinded study was done prospectively for evaluation of the degree of pulpal anaesthesia acquired with conduction blockade of the inferior alveolar nerve (IAN) that was frequency-dependent. Materials and Method: 80 adult subjects (divided into 2 groups of 40 participants each serving their own control) who volunteered for the research proposal randomly received 2 IAN blocks: 1) An IAN block which was then continuously stimulated electrically for 3 minutes for the $1^{\text {st }}$ molar or lateral incisor for 6 cycles over a time period of 64 minutes; 2 ) An IAN block which was followed by mock electrical stimulation for 6 cycles for 64 minutes. The IAN blocks were given at two separate appointments spaced at least 7 days apart in a crossover design. Anesthesia of the first molar and lateral incisor was tested with the help of electric pulp tester. Anesthesia was regarded as successful when 2 consecutive 80 readings were acquired within 15 minutes, and the 80 reading was calibrated in the 60 th minute. Results: The anesthetic success rate for the stimulated IAN block was $35 \%$ for the lateral incisor and $48 \%$ for first molar. For the IAN given mock stimulation, success percent was $18 \%$ for the lateral incisor while it was found to be $62 \%$ for the first molar. The two IAN block techniques showed no significant difference between them. Conclusions: Thus it was concluded that the nerves stimulated in the presence of frequency-dependent local anesthesia did not statistically increase the success rate of pulpal anesthesia for an IAN block.

Keywords: Frequency-dependent nerve block, inferior alveolar nerve block, lidocaine, local anesthesia.

Copyright $\odot 2021$ The Author(s): This is an open-access article distributed under the terms of the Creative Commons Attribution 4.0 International License (CC BY-NC 4.0) which permits unrestricted use, distribution, and reproduction in any medium for non-commercial use provided the original author and source are credited.

\section{INTRODUCTION}

Successful pulpal anesthesia is not always obtained by administering the inferior alveolar nerve (IAN) block [1]. $10 \%$ to $39 \%$ failure rates have been reported through various studies [1-5]. Endodontic clinical studies [2, 3] have estimated success rates between 15-57 \% with the IAN block. Therefore, it would be beneficial to improvise the rate of success of the IAN block. High-frequency electrical stimulation provided repeatedly potentiates local anesthetic action $[4,5]$. The same action is referred to as use-dependent, frequency-dependent, or phasic blockade $[4,6]$. Frequencies of higher magnitude $(100 \mathrm{~Hz})$ of stimulation have greater use-dependent inhibition than those of lower magnitude $(40 \mathrm{~Hz})[4,6]$.
Unfortunately the available data from in vivo studies of frequency-dependent blockade is limited [7, 8]. Stevens et al., [7] found that the onset of anesthesia is accelerated by non-noxious high frequencies electrical stimulation extending sensory blockade spread for the ulnar nerve. Watson et al., [8] showed use- dependent conduction blockade of the median nerve in carpal tunnel syndrome patients. However, no established mechanism has shown how other nerves get affected by use-dependent blockade. Nerve anatomy, its physiology, speed of conduction, and stimulation differs which may also affect the clinical efficacy of usedependent blockade.

Till date no in vivo studies of the IAN block have been conducted using phasic blockade. The aim of 
Jyoti Kiran et al., Sch J Dent Sci, Mar, 2021; 8(3): 89-93

this randomized single-blind prospective study was to investigate the level of pulpal anesthesia obtained with frequency-dependent conduction blockade of the Inferior Alveolar Nerve.

\section{MATERIALS AND METHOD}

80 adult volunteers participated in this single bind randomized study. All of the subjects were in good systemic health as determined by a written case history and questioning done orally. Exclusion criteria included: subjects less than 18 years or more than 65 years of age, any known allergies to local anesthetic agents or sulfites, pregnant or lactating females, any relevant medical history, under any medications that might affect anesthetic assessment (e.g., opioids, antidepressants, analgesic medications and alcohol), any active sites of pathosis in area of interest injection, or inability to give informed written consent. The volunteers received two IAN blocks randomly:

1. An IAN block which was then continuously stimulated electrically for 3 minutes for the $1^{\text {st }}$ molar or lateral incisor for 6 cycles over a time period of 64 minutes;

2. An IAN block which was followed by mock electrical stimulation for 6 cycles for 64 minutes.

The IAN blocks were given at two separate appointments spaced at least 7 days apart in a crossover design. A total of 160 total injections were administered with the crossover design, and each subject was his/her own control. Anesthesia of the first molar and lateral incisor was tested with the help of electric pulp tester. Anesthesia was regarded as successful when 2 consecutive 80 readings were acquired within 15 minutes, and the 80 reading was calibrated in the 60 th minute.

The same side chosen randomly for the 1st injection was also used for the second injection. The canine of the contralateral side was considered as the unanesthetized control to check that the pulp tester was operating properly and that the subject was responding appropriately during each experimental portion of the study. Clinical examinations indicated that all teeth were caries free, no large restorations, and no periodontal disease; none had histories of trauma or sensitivity.

Before the experiment, the two nerve blocks, with stimulation or mock stimulation, were assigned random numbers. Each subject was randomly assigned to the right or left side. The order of assignment to the stimulation or mock stimulation group was also randomly determined.

All stimulation was administered with a modified electric pulp tester. We modified the electric pulp tester by changing the circuitry and adding a twoposition switch. One switch caused a gradual increase in voltage that could be halted by the subjects depressing a hand-held button. However, once the level of stimulation was achieved, this level of stimulation was continually applied at this position. High-frequency $(100 \mathrm{~Hz})$ stimulation is more strongly supported for frequency- dependent conduction block. Therefore, the frequency of stimulation was set at $100 \mathrm{~Hz}$ and verified using a Tektronix oscilloscope. The voltage and current were controlled in the current experiment because the Kerr Analytic Technology pulp tester has a voltage range of 0 to $350 \mathrm{~V}$ and a range of 0 to $50 \mathrm{~mA}$. Only the Hertz (frequency of a current waveform) was modified with the pulp tester to alternate at 100 times each second (based on the experimental studies of frequencydependent blockade). Therefore, Hertz is a measure of how quickly the voltage and current changes or vibrates (100 times a second). The modified pulp tester could also be used as a normal pulp tester when the second position of the switch was activated.

A pilot study using 10 additional subjects tested the ability of the nerve to adapt to stimulation and remain adapted after cessation of the stimulation. The right and left mandibular first molar and right and left lateral incisor were tested in 10 subjects using the modified pulp tester in the stimulation mode with the subject controlling stimulation level with the handheld button. The teeth were stimulated with $100 \mathrm{~Hz}$ for 3 minutes without anesthetic administration to determine if any changes in pulp test readings occurred because of the stimulation when compared with baseline values. After the first minute of stimulation the subject was questioned regarding any change in sensation (higher, same, or less). The subject was allowed to rest for 60 seconds, and three trials were performed at 2-minute intervals. This cycle of stimulation was repeated three times for each molar and lateral incisor.

At the beginning of each experimental appointment and before any injections were given, the experimental teeth and control canine were tested three times with the modified pulp tester in its normal mode of operation. After the tooth to be tested was isolated with cotton rolls and dried with gauze, toothpaste was applied to the probe tip, which was then placed midway between the gingival margin and the occlusal or incisal edge of the tooth. The current rate was set at 25 seconds to increase from no output (0) to the maximum output (80). The number at the initial sensation was recorded. Trained research personnel per- formed all preinjection and postinjection tests.

Subjects were administered a standard IAN block using 2\% lidocaine with 1:100,000 adrenaline and a standard aspirating syringe. Lidocaine was chosen because Scurlock et al., (19) and Courtney (21) found that lidocaine was a more potent blocker of sensory nerves during frequency-dependent conduction block than other anesthetic agents tested. After negative aspiration, $1.8 \mathrm{~mL}$ of the anesthetic solution was deposited over a 1-minute time period. A sterile rubber 
Jyoti Kiran et al., Sch J Dent Sci, Mar, 2021; 8(3): 89-93

stopper, previously placed on the 27-G needle, was adjusted to rest flush with the oral mucosa at the completion of the injection. Upon withdrawal of the needle, the distance from the needle tip to the rubber stopper was measured. The subject was asked every minute for 15 minutes if they were experiencing lip numbness. All subjects used for data analysis reported profound lip numbness.

To blind the subject to whether stimulation was used, a mock treatment was used. An experimenter's box with a red light, a standard voltmeter, and an on/off switch was constructed for this study. Turning the on/off switch to the on position activated the red light and voltmeter (the power source was a $9-\mathrm{V}$ battery and was independent of any voltage applied to the subject's teeth). The box was also wired to the modified pulp tester so that when the switch was activated there was no current output to the pulp tester. Subjects and the pulp testers were told that the voltmeter reading would determine the amount of current delivered to the tooth and they may feel pain. During this time, the subject actually received no stimulation. The experimenter's box was initially shown to the subject but was positioned out of view for the subject during the experimental phase of the study. The stimulation cycle was the same as for the experimental procedure.

At 5 minutes after each block was given, a pulp test reading was recorded. At 6 minutes, the pulp tester probe tip was applied to the test tooth, and the modified pulp tester (stimulation mode) became activated as seen by the advancing digital readout. Once a sensation in the tooth was experienced, the subject halted the advancement in stimulation by depressing the handheld button. Stimulation at the halted level continued for 3 minutes. If no sensation was recorded by the subject, the maximum current ( 80 reading) was applied for 3 minutes. For the mock stimulation, the switch on the experimenter's box was turned on, and the probe was placed on the tooth for 3 minutes. After the stimulation or mock stimulation, the subject was allowed to rest for 1 minute.
The modified pulp tester was switched to the normal mode of operation, and the experimental tooth (lateral incisor or first molar) was tested each minute for 3 minutes. The contralateral control canine was tested 1 minute later. The 3 minutes of continuous stimulation or mock stimulation cycles were then repeated as outlined previously.

Obtained data were analyzed statistically. All subjects used for data analysis had profound lip anesthesia. Comparisons of the two nerve blocks for anesthetic success were analyzed using the McNemar test.

\section{RESULTS}

A total of 80 adult subjects, 35 women and 45 men aged 18 to 36 years with an average age of 25 years, participated in the experimental portion of the study. Forty adult subjects, 20 women and 20 men aged 19 to 38 years with an average age of 25 years, participated in the first molar experiment. Forty adult subjects, 15 women and 25 men, aged 18 to 38 years with an average age of 27 years participated in the lateral incisor experiment. Ten adult males, aged 22 to 38 years with an average age of 26 years, participated in the pilot study.

The pilot study indicated no significant difference between pulp test readings pre and post stimulation with $100 \mathrm{~Hz}$ for 3 minutes without anesthetic administration. Sensation during this stimulation for the first molars and lateral incisors stayed the same in $88 \%$ to $89 \%$ of the subjects, decreased in $4 \%$ to $9 \%$, and increased in $3 \%$ to $5 \%$. Eight subjects in the lateral incisor group $(8 / 88$ injections) and seven subjects in the first molar group (7/87 injections) did not achieve lip numbness with the IAN block. Therefore, the incidence of missed blocks was $8.6 \%$ (15/175 total injections). None of the subjects achieved complete pulpal anesthesia. These 15 subjects were reappointed (13 subjects) or replaced with new subjects (two subjects).

One hundred percent of the 80 subjects used for data analysis had profound lip anesthesia. Anesthetic success is presented in Table-1.

Table-1: Percentages and Number of Subjects Who Experienced Anesthetic Success with the Stimulated IAN Block and the Mock-stimulated IAN Block

\begin{tabular}{|l|l|l|}
\hline \multirow{2}{*}{} & Nerve block \\
\cline { 2 - 3 } & Stimulated IAN (\%) & Mock-stimulated IAN (\%) \\
\hline Lateral incisor* & $35(14 / 40)$ & $18(7 / 40)$ \\
\hline First molar* & $48(19 / 40)$ & $62(25 / 40)$ \\
\hline $\begin{array}{l}\mathrm{n}=40 \text { per group. } \\
* \text { There was no significant difference between the two nerve blocks. }\end{array}$ \\
\hline
\end{tabular}

Figures 1 and 2 present the incidence of pulpal anesthesia (percentage of 80 readings) for the two nerve blocks. There were no statistically significant differences between the two nerve blocks. The mean depth of needle placement to the target site for the IAN 
block was $18 \mathrm{~mm}$ for the stimulated session and $18 \mathrm{~mm}$ for the unstimulated session.

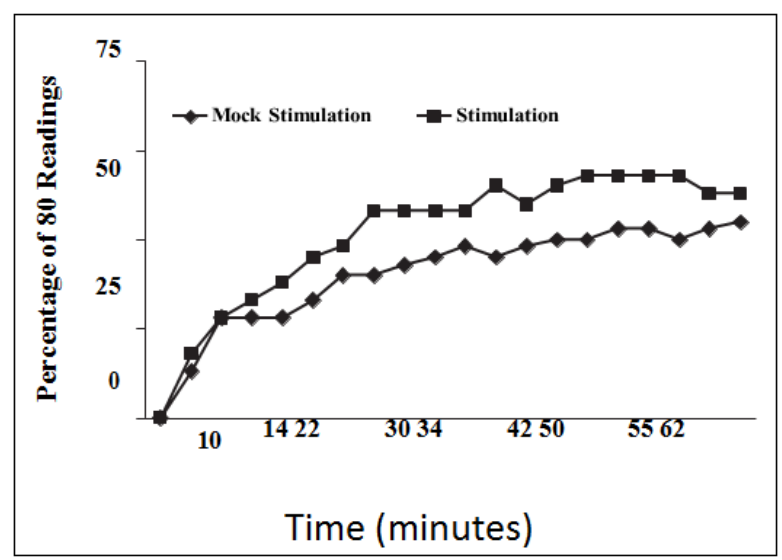

Fig-1: The lateral incisor anesthesia determined by the lack of response to electrical pulp testing at the maximum setting (percentage of $80 / 80 \mathrm{~s}$ ) at each post-injection testing interval for the stimulated and mock stimulated IAN blocks

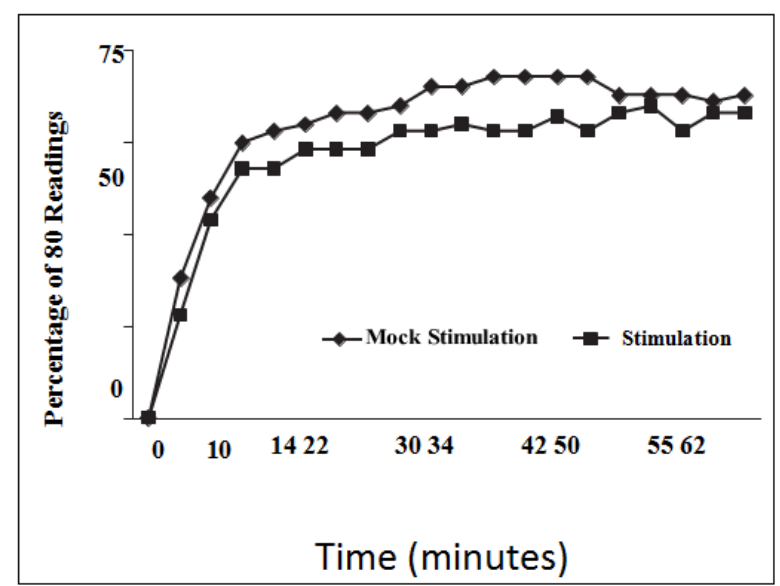

Fig-2: The first molar anesthesia as determined by the lack of response to electrical pulp testing at the maximum setting (percentage of 80/ 80s) at each post-injection testing interval for the stimulated and mock stim ulated IAN blocks

\section{DISCUSSION}

The potential of sodium channel blocking of local anesthetics is dependent on the channel states (i.e., resting state, open state, and inactive state) [9]. Open channel blockade is higher than resting channel blockade [9]. Inactive channel binding seems to be greater than that of resting channel binding. An excitable membrane firing more rapidly will spend longer time in high affinity open and inactive channel states [9]. As a result there is an increase in drug blocking potency. This action is called frequencydependent or use-dependent blockade. The prerequisites for nerve blockade i.e. frequency-dependent include a local anesthetic and a cycle of repetitive stimuli [6].

We anticipated that success rate would be improved by the non-noxious electrical stimulation of specific nerves at high frequency $(100 \mathrm{~Hz})[6,9]$ and in the presence of lidocaine. Placing sodium channels in open-state anesthetic molecules should block ion exchange more readily, thereby improving blockade. Although recordings in the present study would simulate other studies using pulp testing to evaluate anesthesia, constant electrical stimulation in 3-minute cycles was unique to this study. Based on earlier studies $[6,9]$, we believed that experimentally using stimulation in cycles of 3 minutes over the 64 minutes should have resulted in opening of the sodium channels because high levels of stimulation were used to ensure that impulses would be transmitted in partially or incompletely anesthetized nerves. Although no statistically significant differences in anesthetic success was seen in the present study (Table-1), the electrically stimulated block did have higher incidences of 80 readings than the mock stimulated block for the lateral incisor (Fig-2). In accordance with Fink and Cairns [6] who used an animal model, if too many axons are blocked effectively by a local anesthetic agent, a usedependent blockade is difficult to exhibit. Because the lateral incisor had an overall lower incidence of 80 readings than the first molar (Fig-2), indicating less effective blockade of nerve, probably the effects of frequency-dependent blockade would be more apparent in the lateral incisor. However, to be clinically meaningful, we would require a complete set of 80 readings across the 60 minutes so that the patient felt no pain. Further study may be indicated using different stimulation times or anesthetic agents to exhibit frequency- dependent effects more effectively.

Although earlier clinical studies [7, 8] have shown an frequency-dependent blockade effect of local anesthetic, both studies used cutaneous stimulation at a maximum of 30 and $50 \mathrm{~Hz}$, one study used only seven subjects [7], and the other study [8] used patients having an entrapment neuropathy. These studies are different than using normal subjects with noninflamed teeth and stimulating pulpal nerves at $100 \mathrm{~Hz}$ rather than cutaneous stimulation. The limitations of these mentioned studies [7, 8] would indicate that further investigations are necessary to definitively authenticate the effect of frequency-dependent blockade in medicine.

\section{CONCLUSION}

Thus it was concluded that the nerves stimulated in the presence of frequency-dependent local anesthesia did not statistically increase the success rate of pulpal anesthesia for an IAN block.

\section{REFERENCES}

1. Nusstein J, Reader A, Beck M. Anesthetic efficacy of different volumes of lidocaine with epinephrine for inferior alveolar nerve blocks. Gen Dent. 2002;50:372-5. 
2. Cohen HP, Cha BY, Spangberg LSW. Endodontic anesthesia in mandibular molars: a clinical study. J Endod. 1993;19:370-3.

3. Reisman D, Reader A, Nist R, Beck M, Weaver J. Anesthetic efficacy of the supple- mental intraosseous injection of $3 \%$ mepivacaine in irreversible pulpitis. Oral Surg Oral Med Oral Pathol Oral Radiol Endod. 1997;84:676-82.

4. Courtney KR, Kendig JJ, Cohen EN. Frequencydependent conduction block: the role of nerve impulse pattern in local anesthetic potency. Anesthesiology. 1978;48: 111-7.

5. Fink BR, Cairns AM. Differential use-dependent (frequency-dependent) effects in single
Jyoti Kiran et al., Sch J Dent Sci, Mar, 2021; 8(3): 89-93 mammalian axons: data and clinical considerations. Anesthesiology. 1987; 67:477-84.

6. Strichartz GR. The inhibition of sodium currents in myelinated nerve by quaternary derivatives of lidocaine. J Gen Physiol. 1973;62:37-57.

7. Stevens MF, Klement W, Lipfert P. Conduction block in man is stimulation frequency dependent. Anaesthesist. 1996;45:533-7.

8. Watson BV, Brown WF, Doherty TJ. Frequencydependent conduction block in carpal tunnel syndrome. Muscle Nerve. 2006;33:619-26.

9. Courtney KR. Structure-activity relations for frequency-dependent sodium channel block in nerve by local anesthetics. J Pharm Exp Ther. 1980;213:114-9. 